Conclusion Although useful as diagnostic biomarkers, further studies are required to evaluate these potential biomarkers longitudinally and to assess their reliability as surrogates for underlying neurodegenerative changes and clinical disease severity. DTI showed the most promise as a biomarker for disease severity in HSP.

\section{ACUTE LUMBOSACRAL PLEXOPATHY AFTER BLOOD LOSS IN A PATIENT WITH BILATERAL COMMON ILIAC ARTERY OCCLUSION}

Matthew Katz*, Pamela McCombe. Royal Brisbane and Women's Hospital, Brisbane, QLD, Australia

10.1136/jnnp-2019-anzan.42

Introduction We present a case of a 56 year old male with bilateral, asymmetric leg weakness from ischemic lumbosacral plexopathy. This followed acute blood loss from an episode of self-harm, which occurred in the setting of chronic thrombotic occlusion of bilateral common iliac artery (CIA) stents.

Methods A detailed history, examination and neurophysiology study were performed in addition to reviewing case notes and results of other investigations. A brief literature review was also completed.

Results The patient presented with acute blood loss following self-inflicted lacerations to his anterior neck and both wrists. He had asymmetric flaccid, areflexic weakness of his legs with decreased sensation over the right foot and absent distal leg pulses. Magnetic resonance imaging (MRI) of brain and whole spine was normal. Computed tomography angiogram (CTA) revealed significant distal aortic atherosclerotic disease with complete occlusion of bilateral CIA stents. The patient underwent endovascular re-stenting of both common iliac arteries with a good angiographic result. An electromyogram performed one month later was supportive of a lumbosacral plex-opathy, mainly on the right.

Only three cases of acute ischemic lumbosacral plexopathy have been reported in the setting of aorto-iliac occlusive disease. $^{1-3}$

Conclusion Ischemic injury to the lumbosacral plexus is rare, especially outside the surgical setting as seen in this case. It is important to consider this cause in any patient presenting with rapid onset asymmetric, bilateral leg weakness as early recognition and reperfusion may prevent further damage and reduce long-term disability.

\section{REFERENCES}

1. Schreuder AH, Fennis TF, Teijink JA, Koehler PJ. Lumbosacral plexopathy associated with aortoiliac occlusive disease. J Neurol. 2007;254(6):803-5.

2. Chhetri SK, Lekwuwa G, Seriki D, Majeed T. Acute flaccid paraparesis secondary to bilateral ischaemic lumbosacral plexopathy. QJM. 2013;106(5):463-5.

3. Yun S. Ischaemic lumbosacral plexopathy following an acute thrombosed abdominal aortic aneurysm mimicking stroke: A case report. Hong Kong Journal of Emergency Medicine. 2016;23(2):52-6.

\section{HARDING'S DISEASE: AN IMPORTANT MS MIMIC}

${ }^{1}$ Stuti Joshi*, ${ }^{2}$ Allan Kermode. ${ }^{1}$ Sir Charles Gairdner Hospital, Stirling, WA, Australia; ${ }^{2}$ Perron Institute, Sir Charles Gairdner Hospital, Nedlands, WA, Australia

10.1136/jnnp-2019-anzan.43

Introduction Leber's hereditary optic neuropathy is a mitochondrially-inherited disorder characterized by bilateral, painless visual loss, which leads to severe optic atrophy. ${ }^{1}$ LHON can be associated with an MS-like illness referred to as Harding's disease. ${ }^{2}$

We report two siblings, who both harbour the 11778 mtDNA mutation, but manifest markedly different clinical phenotypes; a male with classical LHON and a female with Harding's disease.

Methods and Results A 61-year-old female, who was diagnosed with MS 22 years ago was referred to our service for a second opinion. She developed unilateral painless visual loss in her 20's, was diagnosed with optic neuritis and treated with corticosteroids with some recovery. A second episode of more severe visual loss at age 39 left her with visual impairment to less than finger counting. 4 years later, she had an episode of dysarthria and gait ataxia. MRI showed multifocal white matter lesions involving the juxta-cortical and periventricular regions, cerebellar peduncle and cervical cord. Targeted views of the optic pathways showed hyperintensity of the left optic nerve, with involvement extending into the optic canal.

The patient has one brother who was diagnosed with LHON at age 37 after presenting with severe painless bilateral sequential visual loss. Genetic testing of the index patient confirmed the presence of the same mutation identified in her brother.

Conclusion LHON and Harding's disease demonstrate a great degree of variability in clinical phenotype and penetrance between males and females as well as individuals within the same family. ${ }^{3}$ While there is no evidence for screening MS cohorts for the LHON, consider genetic testing in patients with severe and persistent bilateral visual loss or with a suggestive family history. 4

\section{REFERENCES}

1. Harding $A E$, Sweeney MG, Miller DH, Mumford CJ, Kellar-Wood H, Menard D, McDonald WI, Compston DA. Occurrence of a multiple sclerosis-like illness in women who have a Leber's hereditary optic neuropathy mitochondrial DNA mutation. Brain. 1992 August;115 ( Pt 4):979-89.

2. Palace J. Multiple sclerosis associated with Leber's Hereditary Optic Neuropathy. J Neurol Sci. 2009 November 15;286(1-2):24-7. Review.

3. Pfeffer G, Burke A, Yu-Wai-Man P, Compston DAS, Chinnery PF. Clinical features of MS associated with Leber hereditary optic neuropathy mtDNA mutations. Neurology. 2013;81(24):2073-2081.

4. Yu-Wai-Man P, Chinnery PF. Leber hereditary optic neuropathy. In: Pagon RA MP, Adam Ardinger HH eds. GeneReviews. Seattle, WA: University of Washington, Seattle; 2013. Accessed May 7, 2018.

\section{REAL WORLD EVIDENCE (RWE) ON IMPACT OF AGE ON LONG-TERM PERSISTENCE TO DISEASE MODIFYING THERAPIES (DMTS) IN RELAPSING-REMITTING MULTIPLE SCLEROSIS (RRMS) IN AUSTRALIA}

${ }^{1}$ Andrew Kornberg* ${ }^{2}$ Tim Spelman, ${ }^{3}$ Mark Schulz, ${ }^{3}$ Birendra Arora, ${ }^{4}$ Eric Chung ${ }^{5}$ Prabjot Juneja, ${ }^{3}$ Robert Walker, ${ }^{6}$ Anneke van der Walt, ${ }^{6}$ Helmut Butzkueven. ${ }^{1}$ Royal Children's Hospital, Parkville, VIC, Australia; ${ }^{2}$ Department of Medicine, University of Melbourne, Melbourne, VIC, Australia; ${ }^{3}$ Novartis, North Ryde, NSW, Australia; ${ }^{4}$ Prospection Pty Ltd, Australian Technology Park, Eveleigh, NSW, Australia; ${ }^{5}$ Prospection Pty Ltd, Australian Technology Park, Eveleigh, NSW, Australia; ${ }^{6}$ MS and Neuroimmunology Group, Central Clinical School, Monash University and Alfred Hospital, Melbourne, VIC, Australia

\subsection{6/jnnp-2019-anzan.44}

Background Age has been independently associated with higher rates of treatment discontinuation in multiple sclerosis.

Objective The current study examines the impact of age on persistence for all reimbursed DMTs for RRMS in Australia. Methods The Pharmaceutical Benefits Scheme (PBS) 10\% sample supplied by the Department of Human Services was used 